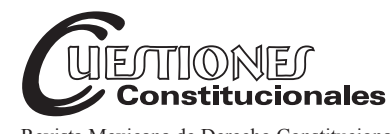

Revista Mexicana de Derecho Constitucional Núm. 43, julio-diciembre 2020

\title{
El amparo judicial y la reforma al sistema de justicia en México (1987-2018)
}

\author{
Judicial amparo and the judicial reform in Mexico
}

(1987-2018)

\begin{abstract}
Alberto Abad SuÁREZ ÁviLA*
RESUMEN: El artículo parte de una pregunta relevante para el constitucionalismo mexicano. Después del intenso periodo de tres décadas de reformas al sistema de justicia ¿cuál es la función principal que tiene el amparo judicial hoy? Para dar respuesta a esta pregunta, se plantea una metodología sociojurídica observando dos variables: 1) la modificación en gran escala al diseño institucional del sistema de justicia, y 2) la modificación al amparo judicial específicamente. La hipótesis principal del artículo es que el proceso de reformas al sistema de justicia tuvo un impacto profundo en las nuevas funciones del juicio de amparo judicial, debido a que trajo consigo una amplia miscelánea de nuevos procesos para resolver conflictos, incluso de debido proceso y otras violaciones constitucionales. La conclusión del artículo es que, desde el análisis del diseño constitucional, se aprecia que la reforma judicial ratificó la función contro-

ABSTRACT: This paper departs from a crucial question to Mexican constitutionalism. After an intense period of three decades of judicial system reform, what is today's Judicial Amparo main role? To answer the former, the author proposes a sociolegal methodology, analyzing two variables: 1) the impact big scale constitutional reform on the system of justice has over Judicial Amparo, and 2) the constitutional reform to the Judicial amparo itself. The main hypothesis of this paper is that both variables are extremely important to understand today's role of Judicial Amparo, due to the enaction of new means of protection and conflict resolution, that could have a relevant effect on due process and other constitutional violations. The paper concludes that the judicial reform ratifies the controlling of the judicial system as the main function of the Judicial Amparo, over other expectations built during the previous years.
\end{abstract}

* Investigador de tiempo completo del Instituto de Investigaciones Jurídicas de la UNAM. Miembro del Sistema Nacional de Investigadores Conacyt, México. Correo: abad@unam.mx ORCID: 0000-0002-0809-3534. El autor agradece las observaciones y recomendaciones recibidas por dos dictámenes anónimos a una versión anterior a este trabajo, así como los comentarios recibidos a esta versión final de Héctor Fix-Fierro y Dulce María Milán Rodríguez. 
ladora del sistema de justicia como la principal del amparo judicial, por encima de otras expectativas construidas en los años previos a su concreción.

Palavras chave: amparo judicial, reforma constitucional, justicia.
Keywords: judicial protection, constitutional reform, justice.

\begin{abstract}
SUMARIO: I. Análisis sociojurídico del proceso de reforma al sistema de justicia en México. De la justicia de elite a la justicia ordinaria (1987-2018). II. La reforma al amparo y sus implicaciones para el amparo judicial. III. Conclusiones. IV. Bibliografía.
\end{abstract}

La intención de este artículo es analizar la función que tiene hoy el amparo judicial en el sistema de justicia en México, tomando como referencia el proceso de rediseño institucional resultado de tres décadas de intensas reformas constitucionales. El amparo judicial es pieza central de la organización y funcionamiento de los poderes judiciales federal y locales por el sistema de doble jurisdicción que mantiene el federalismo mexicano. ${ }^{1}$ A través de esta figura, el Poder Judicial de la Federación puede resolver en última instancia cualquier caso presentado ante el sistema de justicia del país. Por lo mismo, es un tema que genera grandes tensiones entre diversos sectores jurídicos, que, en los extremos, se dividen entre quienes expresan una absoluta dependencia hacia su permanencia dentro del sistema de justicia como garantía de esta última y quienes esgrimen una aguda crítica por el poder que otorga a la jurisdicción federal a costa del derecho local.

Siendo ésta una discusión de varias décadas en el sistema jurídico mexicano, el artículo busca actualizar lo que ha pasado con ella, después del intenso proceso de reformas constitucionales, para conocer en dónde se encuentra la función principal del amparo judicial dentro del sistema de justicia al día de hoy. En un artículo escrito a principios de este siglo intitulado "El futuro del amparo judicial", Héctor Fix-Fierro pronosticaba seis caminos que podría seguir esta institución: el mantenimiento del statu quo, limitar la procedencia del amparo judicial, mejorar la eficiencia y la calidad

\footnotetext{
1 Fix-Fierro, Héctor, "El futuro del amparo judicial”, Bien Común y Gobierno, año 7, núm 81, septiembre, 2001, p. 5

Esta obra está bajo una Licencia Creative Commons

Atribución-NoComercial-SinDerivar 4.0 Internacional, IIJ-UNAM.
} 
de los tribunales ordinarios, crear supremas cortes o tribunales de casación locales, mejorar la formación y el ejercicio profesional de los abogados o cambiar radicalmente el modelo de organización judicial. ${ }^{2}$ En mi opinión, todos estos temas se mantuvieron en tensión durante los años previos a la reforma constitucional de 2011.

Para responder al objetivo del artículo, el tema se aborda desde el análisis sociojurídico del proceso de reformas constitucionales, observando: 1) cómo se modificó el sistema de justicia a lo largo de este periodo, y 2) cómo se modificó el diseño institucional del amparo judicial específicamente. La finalidad de esta metodología es conocer cómo ha interactuado la reforma constitucional en estos dos niveles para proveer un nuevo diseño institucional del sistema de justicia en general, y de esta manera concluir cuál es el espacio y funciones que se le otorgan al amparo judicial en la actualidad. La hipótesis principal de este artículo es que el cambio en el sistema de justicia tiene un impacto muy importante en el lugar que finalmente el proceso de reformas dará al amparo judicial.

El artículo establece como conclusión principal que el proceso de reforma constitucional corroboró la función controladora que tiene el amparo judicial sobre el sistema de justicia, ratificándolo como el elemento unificador del sistema de justicia de doble jurisdicción, sin resolver muchos de los problemas que lo aquejan desde hace tiempo. A pesar de que muchas expectativas estuvieron puestas en que el amparo judicial se ampliara para reconocer competencia a los tribunales locales, o para flexibilizar su acceso, parte de la conclusión de este texto es que muchas de esas expectativas ya podrían estar cubiertas en el diseño institucional, por la miscelánea de opciones de solución de conflictos jurisdiccionales y no jurisdiccionales que el proceso de reforma constitucional ha traído.

El artículo se organiza en tres partes, de la siguiente manera: en la primera, se presenta la explicación del proceso de reforma al sistema de justicia en México de 1987 a 2018, dividido en dos momentos, el de la justicia de elite (1987-2006), y el de la justicia ordinaria (2006-2018). En la segunda parte se analiza la reforma al amparo de 2011 en relación con el amparo judicial. Finalmente, se incluye un breve apartado final, en donde se presentan las conclusiones del estudio mediante la conexión entre las reformas que ha sufrido el sistema de justicia y el amparo judicial.

\footnotetext{
2 Ibidem, pp. 11-16.
} 


\section{ANÁLISIS SOCIOJURÍDICO DEL PROCESO DE REFORMA} AL SISTEMA DE JUSTICIA EN MÉXICO. DE LA JUSTICIA DE ELITE A LA JUSTICIA ORDINARIA (1987-2018)

El más reciente proceso de reformas al sistema de justicia en México inició en 1987, como parte de un movimiento internacional de transformación, que se conoce como la expansión global del Poder Judicial en el mundo, ${ }^{3}$ con un desarrollo significativo en la región latinoamericana. ${ }^{4}$ El proceso de reforma en México se ha llevado paulatinamente a lo largo de tres décadas, a diferencia de lo acontecido en otros países de América Latina, que modificaron sus instituciones de forma concentrada, mediante la provisión de nuevas Constituciones, nuevos tribunales y nuevos procesos, en periodos más cortos. Como resultado de este proceso, se ha modificado el sistema de justicia en prácticamente todos sus aspectos, desde el rediseño institucional del Poder Judicial, fiscalías y defensorías, federal y locales; el establecimiento de nuevos regímenes procesales y medios alternativos de resolución de conflictos hasta, incluso, la modificación de los principios procesales fundamentales. Lo más relevante que puede decirse del proceso de reforma en México es que ha sido muy profundo, al modificar completamente las funciones, la ubicación y la distribución de poder dentro del sistema de justicia.

3 Tate, C. Neal and Vallinder, Torbjörn (eds.) (1995), The Global Expansion of Judicial Power, New York, New York University Press.

4 Helmke, Gretchen y Rios Figueroa, Julio, Courts in Latin America, New York, Cambridge University Press, 2010. De acuerdo con Linn Hammergren, son cinco las principales características del proceso de reforma judicial en América Latina: “1) surgen después de largas décadas durante las cuales los poderes judiciales sufrieron una inusual falta de atención; 2) aunque los cambios legales constituyen un elemento central de las reformas también se incorporaron elementos novedosos como la creación de nuevas organizaciones sectoriales, inversiones en equipos y edificios, una nueva atención hacia la administración y la gestión, así como un énfasis en valores como el mayor acceso a la justicia, su independencia y eficacia; 3) las reformas han sido financiadas, y en muchos casos iniciadas por donadores de agencias internacionales y un grupo muy diverso de patrocinadores locales; 4) el proceso se ha beneficiado de un inusual nivel de contacto e intercambio entre los países participantes. Esto ha ayudado a vencer las resistencias locales al cambio y ha acelerado la adopción de nuevas tendencias a través de los países. 5) La reforma judicial se encuentra cada vez más vinculada a amplios objetivos sociales, incluyendo el crecimiento económico, la lucha contra la pobreza y la democratización". Hammergren, Linn, "Usos de la investigación empírica para el reenfoque de las reformas judiciales. Lecciones desde cinco países en América Latina”, Hoy, abril, año/vol. 39, Salamanca, Universidad de Salamanca, 2005. p. 16. 
Para analizar el proceso de reformas constitucionales al sistema de justicia, se analiza el periodo de 1987 a 2018 en dos momentos. Al primer momento le llamo la reforma a la justicia de elite (1987-2000), debido a que se concentró en la modificación de las estructuras judiciales y en la provisión de medios procesales para permitir que el Poder Judicial de la Federación pudiera convertirse en el resolutor de conflictos entre actores poderosos. Al segundo momento lo nombro la reforma a la justicia cotidiana (2000-2018), caracterizado por enfocarse en la modificación de medios procesales para resolver conflictos cotidianos. ${ }^{5}$

\section{Primera etapa (1987-2000): la justicia de elite}

El primer conjunto de reformas constitucionales al sistema de justicia (1987-2000) se orientó a la construcción de un nuevo perfil de autonomía institucional del Poder Judicial para contribuir a la transición democrática en el país, así como a proveer medios de resolución de conflictos para actores políticos relevantes. ${ }^{6} \mathrm{Si}$ hacemos una similitud con otros procesos sociales, estas fueron reformas hechas a los aspectos macro de la justicia. No sólo se modificó el Poder Judicial de la Federación, sino que, dada la naturaleza de doble jurisdicción de nuestro país, también se modificó el diseño institucional de los poderes judiciales locales para conseguir su autonomía institucional, aunque esto tuvo menor éxito. ${ }^{7}$

5 Esta propuesta de clasificación en el periodo tendrá que ser debatida a mayor profundidad con los estudiosos de la reforma judicial. Pérez-Perdomo, por ejemplo, prefiere clasificarla en a) reforma judicial, Suprema Corte y amparo, y b) justicia ordinaria, atendiendo a un criterio más bien competencial dentro de la doble jurisdicción mexicana (Pérez Perdomo, Rogelio, Gente del derecho y cultura jurídica en América Latina, México, UNAM, Instituto de Investigaciones Jurídicas, 2013). Héctor Fix-Fierro apunta hacia esta problemática al señalar que el caso de la reforma judicial "se trata de una reforma que ha perseguido objetivos diversos y un tanto erráticos en distintos momentos... ha procedido sin basarse en diagnósticos claros y completos, sin seguir un plan sistemático y sin llevar a cabo una discusión pública suficiente" (Fix-Fierro, Héctor, La reforma judicial mexica$n a$, ¿de dónde viene?, ¿hacia dónde va?, México, UNAM, Instituto de Investigaciones Jurídicas, 2002).

6 Domingo, Pilar, "Rule of Law, Citizenship and Access to Justice in Mexico", Mexican Studies/Estudios Mexicanos, vol. 15, núm. 1, Winter 1999, pp. 151-191.

7 Concha Cantú, Hugo Alejandro y Caballero Juárez, José Antonio, Diagnóstico sobre la administración de justicia en las entidades federativas: un estudio institucional sobre la justicia local en México, México, UNAM, Instituto de Investigaciones Jurídicas- 
La reforma judicial en su primera etapa consiguió importantes resultados en la construcción de mayor autonomía institucional para los poderes judiciales federal y locales, y mejores garantías jurisdiccionales para los juzgadores y otros operadores jurídicos. Ilustrativamente, Héctor FixFierro señalaba que "en México, el Poder Judicial, más que tercer poder, era considerado un poder de tercera, es decir, que no actuaba en relación de paridad con el Ejecutivo y el Legislativo, sino que ocupaba un tercer sitio en términos de autoridad institucional, prestigio social y recursos económicos". ${ }^{8} \mathrm{Al}$ cabo de pocos años de las reformas de 1987 y 1994, el Poder Judicial ocupaba ya un nuevo espacio dentro de la distribución de poderes en México.

Entre las características más relevantes del proceso se encuentran la conversión de la Suprema Corte de Justicia de la Nación en un tribunal constitucional, mediante diversas acciones, tales como la pérdida de competencia originaria sobre el amparo directo hacia los tribunales colegiados de circuito en 1987 y su rediseño institucional en 1994.

Son sumamente importantes también la creación del Consejo de la Judicatura Federal, la adopción de nuevas garantías jurisdiccionales para los integrantes del Poder Judicial, como la carrera judicial. También destaca la incorporación del Tribunal Electoral al Poder Judicial de la Federación. En el aspecto procesal sobresale la incorporación de la acción de inconstitucionalidad como nuevo medio de control de constitucionalidad y la reactivación de la controversia constitucional mediante la ampliación de la legitimación a los municipios.

El hecho de que estas reformas se hayan enfocado en el rediseño del Poder Judicial, así como en la promoción de mecanismos judiciales para resolver controversias entre actores políticos relevantes, me hace denominar a este periodo como de reforma a la justicia de elite. En el lado negativo de esta descripción debe decirse que los conflictos diarios no recibieron atención dentro de este proceso de reformas constitucionales, lo que fortalece la idea de una justicia de elite, centrada en los actores poderosos. Entre los temas que se dejaron de lado se encuentra el juicio de amparo.

National Center for State Courts, 2001. Beer, Caroline C., "Judicial Performance and the Rule of Law in the Mexican States", Latin American Politics and Society, vol. 48, Issue 3 September, 2006.

8 Fix-Fierro, Héctor, op. cit., 2003, p. 251. 
La literatura habla de una serie de razones endógenas y exógenas para explicar la racionalidad de este primer conjunto de reformas. ${ }^{9}$ Dentro de las razones exógenas destaca el mencionado proceso de reforma judicial emprendido en toda la región latinoamericana y en otras regiones del mundo como parte de un fenómeno global de promoción o expansión del Poder Judicial. ${ }^{10}$ La expansión del Poder Judicial en el mundo se da en un contexto en el cual la promoción de una economía de mercado avanza acompañada de la promoción de una agenda de democracia liberal y de un Estado de derecho. ${ }^{11}$

En cuanto a las razones endógenas, para varios autores se trató de un proceso mediante el cual el partido hegemónico estableció en el Poder Judicial un reaseguro para su actuación en un contexto de alternancia..$^{12}$ En lo que parece el conjunto de hipótesis más populares, el énfasis estuvo puesto en la construcción de la Suprema Corte de Justicia de la Nación como un tribunal constitucional capaz de resolver conflictos entre actores políticos relevantes en un contexto de gobiernos divididos, que anteriormente le correspondió resolver al presidente de la República.

Veamos este proceso a detalle. El primer evento que observamos en el proceso de reforma al sistema de justicia se inicia con la reforma constitucional del 10 de agosto de 1987, que tuvo como eje principal la construcción de un perfil de tribunal constitucional en la Suprema Corte de Justicia de la Nación, descargándola de la atención de ordinario de muchos de sus asuntos, principalmente de amparos directos o uniinstanciales, denominados de esta forma justamente porque la jurisdicción de la Suprema Corte se asumía directamente en única instancia. En esta reforma se delegó a los tribunales colegiados de circuito la jurisdicción completa respecto del amparo directo, y se dejó a la Suprema Corte de Justicia su atención exclusivamente mediante la revisión por violaciones directas a la Constitución

\footnotetext{
9 El tema de las razones endógenas y exógenas de la reforma se encuentra muy bien discutido en López Ayllón, Sergio y Fix-Fierro, Héctor, “¡Tan cerca, tan lejos! Estado de Derecho y cambio jurídico en México (1970-2000)", Boletín Mexicano de Derecho Comparado, México, año XXXIII, núm. 97, enero-abril, 2000, pp. 155-267.

10 Tate y Vallinder, op cit.

11 Trubek, David M. y Santos, Alvaro, "Introduction: The Third Moment in Law and Development Theory and the Emergence of a New Critical Practice", en Trubek, David M. y Santos, Alvaro, The New Law and Economic Development A Critical Appraisal, New York, Cambridge University Press, 2011.

12 Rios Figueroa, Julio, "Fragmentation of Power and the Emergence of an Effective Judiciary in Mexico", Latin American Politics and Society, Spring, vol. 49, num. 1, 2007.
} 
y la facultad de atracción. ${ }^{13}$ Además, se modificaron los poderes judiciales locales para garantizar su independencia conforme a la reforma a la fracción III del artículo 116 constitucional. ${ }^{14}$

La reforma constitucional de 1994 es la más trascendental del periodo, y se centró en el rediseño institucional de la Suprema Corte de Justicia de la Nación, así como en la previsión y promoción de nuevas formas de control de constitucionalidad. Entre los cambios más relevantes que introdujo se encuentra la reducción a once ministros en la integración del Pleno de la Suprema Corte. También se fusionaron las cuatro salas que funcionaron anteriormente para quedar dos: Civil-Penal y Laboral-Administrativa. Se estableció una nueva regla de quince años de duración para el encargo de ministro, se eliminó la anterior regla de duración vitalicia en el cargo, y se mejoraron los salarios y las condiciones laborales. En el tema más polémico de la reforma se creó un nuevo medio de control de constitucionalidad abstracto denominado "Acción de inconstitucionalidad", con una restringida legitimación para sujetos públicos relevantes, en términos similares a las acciones abstractas de constitucionalidad, propias de los tribunales constitucionales europeos de la posguerra. ${ }^{15}$ Además de la descarga del trabajo jurisdiccional en los tribunales inferiores, se creó el Consejo de la Judicatura Federal con el objetivo de que la Suprema Corte de Justicia de la Nación pudiera descargar su labor administrativa en esta institución. ${ }^{16}$

13 Decreto por el que ese adicionan la fracción XXIX-H al artículo 73, la fracción I-B, al artículo 104, y un párrafo final a la fracción V del artículo 107; se reforma el artículo 94, los párrafos primero y segundo del artículo 97, el artículo 101, el inciso a) de la fracción III, el primer párrafo y el inciso b) de la fracción V y las fracciones VI, VIII y XI del artículo 107; y se derogan los párrafos segundo, tercero y cuarto de la fracción I del artículo 104 y el segundo párrafo de la fracción IX del artículo 107 de la Constitución Política de los Estados Unidos Mexicanos, Diario Oficial de la Federación, 10 de agosto de 1987, pp. 3 y 4.

14 Decreto por el que se reforman los artículos 17, 46, 115 y 116 de la Constitución Política de los Estados Unidos Mexicanos para quedar en los términos que se indican, Diario Oficial de la Federaciónpp. 3 y 4.

15 Brage Camazano, Joaquín, La acción abstracta de inconstitucionalidad, México, UNAM, Instituto de Investigaciones Jurídicas, 2005.

16 Decreto mediante el cual se declaran reformados los artículos 21, 55, 73, 76, 79, $89,93,94,95,96,97,98,99,100,101,102,103,104,105,106,107,108,110,111,116$, 122 y 123 de la Constitución Política de los Estados Unidos Mexicanos. Diario Oficial de la Federación, México, 31 de diciembre de 1994, primera sección, pp. 2-10. 
La primera etapa de reformas concluyó con dos temas importantes. En 1996 se reformó la Constitución de nueva cuenta para incorporar al Tribunal Electoral al Poder Judicial de la Federación. ${ }^{17}$ En 1999 se dio una nueva reforma judicial, que tiene como centro regresar el control del Consejo de la Judicatura Federal a la Suprema Corte de Justicia de la Nación. ${ }^{18}$

Respecto del juicio de amparo en general, su poca presencia en el proceso de reforma al sistema de justicia recibió fuertes críticas. En mi opinión, la más acertada fue la que presentó el profesor Rolando Tamayo, respecto a que no hubo claridad de la función del juicio de amparo en el sistema jurídico mexicano por el poder reformador. El proceso reformador se llevó a cabo sin tener en mente la importancia del juicio de amparo en el sistema constitucional mexicano y las características propias de éste, a pesar de que existían ya muchas voces que pugnaban por una profunda reforma del juicio de amparo. ${ }^{19}$

En su lugar, se alejó a la Suprema Corte de Justicia de la Nación de la atención ordinaria del juicio de amparo y se privilegió la atención de los renovados medios de control de constitucionalidad: la acción de inconstitucionalidad y la controversia constitucional. La reforma de 1994 se enfocó en la construcción de un tribunal constitucional kelseniano, más útil para los actores políticos relevantes que para la resolución de conflictos cotidianos. ${ }^{20}$ Durante varios años, el foro jurídico impulsó una modificación constitucional a la materia de amparo como uno de los elementos esenciales para la mejora del sistema de justicia. Pese a ello, hasta 2011,

17 Decreto mediante el cual se declaran reformados diversos artículos de la Constitución Política de los Estados Unidos Mexicanos, Diario Oficial de la Federación, 22 de agosto de 1996, pp. 2-13.

18 Decreto por el que se reforman los artículos 94, 97, 100 y 107 de la Constitución Política de los Estados Unidos Mexicanos, Diario Oficial de la Federación, 11 de junio de 1999, primera sección, pp. 2-4.

19 Tamayo Salmorán, Rolando, "El poder y la judicatura (breve comentario sobre la jurisdicción de amparo y la función judicial)", Boletín Mexicano de Derecho Comparado, núm. 63, México, 1998.

20 Magaloni, Ana Laura, “¿Por qué la Suprema Corte no ha sido instrumento para los derechos fundamentales?", en Ferrer, Eduardo y Zaldívar, Arturo, La ciencia del derecho procesal constitucional. Estudios en homenaje a Héctor Fix-Zamudio en sus cincuenta años como investigador del derecho, México, UNAM, Instituton de Invstigaciones Jurídicas, 2009. 
como se publicó en un popular artículo a mediados de la primera década del siglo, el gran olvidado fue el juicio de amparo. ${ }^{21}$

El amparo judicial jugó un papel secundario en las intenciones del legislador en este periodo de reforma, ya que se relegó a la atención de los tribunales colegiados de circuito para permitir que la Suprema Corte de Justicia de la Nación se concentrara en la atención de nuevos medios de control de constitucionalidad a su disposición. La función residual que se le dejó a la SCJN en el amparo judicial mediante la facultad de atracción fue escasamente utilizada por ella misma. ${ }^{22}$

\section{Segunda etapa: reforma a la justicia ordinaria (2000-2018)23}

En la segunda etapa del proceso de la reforma judicial en México se ha perseguido conseguir un mayor impacto en la justicia ordinaria. Este segundo conjunto de reformas constitucionales se concentró en la atención de conflictos cotidianos, tanto a nivel federal como local. Entre las modificaciones constitucionales más relevantes se encuentran la reforma procesal penal y de medios alternativos de resolución de controversias, ${ }^{24}$ la reforma a las acciones colectivas, ${ }^{25}$ la reforma en materia de amparo ${ }^{26}$

\footnotetext{
21 Magaloni, Ana Laura y Zaldívar, Arturo, "El ciudadano olvidado", Nexos, México, año 28, vol. XXVIII, núm. 342, junio 2006.

22 Entre 1995 y 2002 apenas se rebasó la veintena de solicitudes de facultad de atracción discutidas. Suárez Ávila, op. cit., p. 64.

23 Karina Ansolabehere (2007) habla de las reformas de las reformas judiciales refiriéndose al conjunto de reformas que "que amplían, rectifican, continúan, complementan o retraen las que tuvieron lugar en la década del 90" en América Latina. Las clasifica como relativas a: a) independencia, b) control político, c) eficiencia, d) acceso a la justicia y e) derechos.
}

24 Decreto por el que se reforman y adicionan diversas disposiciones de la Constitución Política de los Estados Unidos Mexicanos, Diario Oficial de la Federación, primera sección, 18 de junio de 2008. Consultado en: http://dof.gob.mx/nota_detalle.php? codigo $=5046978 \&$ fech $a=18 / 06 / 2008$.

25 Decreto por el que se adiciona un párrafo tercero y se recorre el orden de los párrafos subsecuentes del artículo 17 de la Constitución Política de los Estados Unidos Mexicanos, Diario Oficial de la Federación, primera sección, 29 de julio de 2010. Consultado en http://dof.gob.mx/nota_detalle.php? codigo $=5153572 \&$ fecha $=29 / 07 / 2010$.

26 Decreto por el que se reforman, adicionan y derogan diversas disposiciones de los artículos 94, 103, 104 y 107 de la Constitución Política de los Estados Unidos Mexicanos, Diario Oficial de la Federación, primera sección, 6 de junio de 2011. Consultado en http://dof.gob.mx/nota_detalle.php?codigo $=5193266 \&$ fecha $=06 / 06 / 2011$. 
y la de derechos humanos, ${ }^{27}$ así como las iniciativas presidenciales derivadas del proceso de diálogos para la justicia cotidiana. ${ }^{28}$

La característica principal de este segundo conjunto de reformas constitucionales al sistema de justicia es, como se ha mencionado, la provisión de medios procesales para la atención de la justicia ordinaria. Lo anterior tendrá una gran influencia en los alcances de la reforma al juicio de amparo que se presentó en 2011, muchos años después de iniciado el proceso de cambio. Otra característica relevante de la segunda etapa es que muchas de estas modificaciones, previo a alcanzar el nivel constitucional, fueron emprendidas por la justicia local, en una trayectoria de periferia a centro, como la relativa a la materia penal, o a los mecanismos alternativos de solución de controversias. Son reformas de segunda generación, porque gran parte de la probabilidad de su éxito está fundado en el buen funcionamiento de los cambios y principios introducidos en la primera etapa, principalmente en los temas de diseño institucional de los poderes judiciales, como la construcción de autonomía institucional de la Suprema Corte de Justicia de la Nación y la independencia y profesionalización de los juzgadores.

A pesar de que en el inicio de la segunda etapa de modificaciones constitucionales no se dieron reformas en materia de amparo, el proceso tiene una gran deuda con esta materia, ya que fue en derredor de ella donde se detonaron las discusiones más importantes de la reforma al sistema de justicia. Por ello, considero que el punto de inflexión entre la primera y la segunda etapa se da a finales del siglo pasado, cuando la Suprema Corte de Justicia de la Nación realizó procesos de consulta a la ciudadanía para conocer las necesidades en materia de justicia.

El primero de ellos fue la Comisión de Análisis de Propuestas para la Elaboración de un Anteproyecto de Nueva Ley de Amparo, que sesionó entre 1999 y 2000, y que tuvo una importante respuesta de parte del sector académico y de abogados del país. El segundo proceso fue emprendido en 2003 mediante la Consulta Nacional sobre una Reforma Integral y Coherente del Sistema de Impartición de Justicia en el Estado Mexicano,

27 Decreto por el que se modifica la denominación del capítulo I del titulo primero y reforma diversos artículos de la Constitución Política de los Estados Unidos Mexicanos, Diario Oficial de la Federación, primera sección, 10 de junio de 2011. Consultado en: http://dof.gob.mx/nota_detalle.php?codigo $=5194486 \&$ fecha $=10 / 06 / 2011$.

28 Las conclusiones e iniciativas que se presentaron pueden consultarse en https:// www.gob.mx/justiciacotidiana. 
emprendida por la Suprema Corte de Justicia de la Nación, que produciría el Libro Blanco de la Reforma Judicial. ${ }^{29}$

En el Libro Blanco de la Reforma Judicial se encuentra un ambicioso proyecto que paso a paso busca modificar al sistema de justicia en México a través de tres ejes temáticos: reforma al amparo, fortalecimiento de los poderes locales de las entidades federativas y reforma de la justicia penal. Respecto de la reforma del amparo, la presentan como "una condición previa y necesaria, pero no suficiente, para mejorar sustantivamente la justicia del país, pues ésta es la institución que articula y da congruencia al conjunto del sistema". En el Libro Blanco se establecieron cuatro acciones concretas para lograr dichos objetivos:

1) Reformar el amparo mediante modificaciones legislativas, retomando el proyecto de nueva Ley de Amparo elaborado por la Suprema Corte, o incluso elaborando un Código Procesal Constitucional; 2) reformar al amparo a través de la jurisprudencia; 3 ) mejorar la sistematización de la jurisprudencia simplificando su consulta y mejorando la comprensión de sus alcances y efectos, y 4) adoptar medidas de gobierno judicial para la reforma del amparo (por ejemplo, a través de acuerdos generales) (Caballero, Oñate y López Ayllon, 2006, p. 392). ${ }^{30}$

En 2007, la Primera Sala de la Suprema Corte de Justicia de la Nación tomó la iniciativa de incrementar su participación en la protección de los derechos humanos, mediante la facultad de atracción en el juicio de amparo, lo que representa un momento importante en el comportamiento de la SCJN respecto de la materia. La razón de este cambio puede encontrarse en una nueva integración de la SCJN con ministros más activos, así como en el seguimiento de políticas judiciales específicas para ello desde las recomendaciones sugeridas por el Libro Blanco. ${ }^{31}$ Para entonces, las críticas a la ausencia de un trabajo importante de la SCJN en materia de protección de derechos humanos ya eran muy duras (Ibarra, 2007). ${ }^{32}$

29 Caballero Juárez, José Antonio et al., Libro Blanco de la reforma judicial en México, México, SCJN, 2006.

30 Ibidem, p. 392.

31 Suárez Ávila, Alberto Abad, La protección de los derechos fundamentales en la Novena Época de la Suprema Corte, México, Porrúa, 2015.

32 Ibarra Palafox, Francisco, "La Suprema Corte de Justicia y consolidación democrática en México", en Ferrer, Eduardo y Arturo Zaldivar, La ciencia del derecho procesal 
La primera reforma de gran calado a la justicia ordinaria se da en materia penal en 2008. Mediante esta reforma se sustituyó completamente el sistema procesal penal, en ambas jurisdicciones, transformándolo de un sistema inquisitivo a uno acusatorio, adversarial y oral. Se incluyó un apartado A al artículo 20 constitucional para establecer los principios de contradicción, concentración, inmediación y publicidad que regulan las nuevas formas procesales. Se incluyeron nuevos actores, entre ellos, los más importantes, a los jueces de control, intermedios y de audiencia oral; fiscalías; defensorías públicas y asesores jurídicos de la víctima. Se constitucionalizaron el arraigo domiciliario, la duplicidad de términos y otros medios de combate a la delincuencia organizada. ${ }^{33}$

Gran parte de la reforma procesal fue guiada para hacer más eficiente el proceso, al tiempo que también hubo una relevante intención de alinear los procesos penales en México con los paradigmas internacionales de protección de los derechos humanos. Esto último resulta especialmente importante en la relación que guarda con el amparo judicial, debido a que la reforma atiende muchos de los problemas de violación a los derechos procesales establecidos en la Constitución a través de figuras de atención más temprana, como los jueces de control para garantizar la legalidad de las detenciones, investigaciones y actuaciones tempranas del proceso penal o los defensores públicos como garantía de la prevalencia del principio

constitucional. Estudios en homenaje a Héctor Fix-Zamudio en sus cincuenta años como investigador del derecho, t. II, México, UNAM, Instituto de Investigaciones Jurídicas, 2009.

33 Cierto es que previamente se presentaron otras reformas en materia penal, que tuvieron cierta relevancia, pero ninguna como la de 2008. Cabe destacar algunas de ellas, como la reforma de 1993, que modificó los artículos 16, 19, 20 y 119 constitucionales, y que tuvo como orientación una estructura del sistema finalista (2000-2017). La parte más controvertida de la reforma fue la inclusión del concepto elementos del tipo en lugar del tradicional cuerpo del delito. En 1994 se suprimió el monopolio del ejercicio de la acción penal. En 1996 se constitucionalizó el combate a la delincuencia organizada. En 1999 se reformaron los artículos 16, 19, 22 y 123 para retomar el concepto de cuerpo del delito y la probable responsabilidad del indiciado. En 2000 se reformó la Constitución para establecer las garantías de la víctíma u ofendido del delito, y en 2005 se reconoció formalmente la jurisdicción de la Corte Penal Internacional y la supresión de la pena de muerte. Véase Suarez Ávila, Alberto Abad, "La reforma constitucional en materia penal en México 1993-2008. Una explicación desde la sociología jurídica”, en Luis René Guerrero Galván y Carlos María Pelayo Moller (coords.), 100 años de la Constitución mexicana: de las garantías individuales a los derechos humanos, México, UNAM, Instituto de Investigaciones Jurídicas, 2016. 
de contradicción. Previo a la reforma de 2008, las violaciones al debido proceso eran revisables en el sistema de justicia mexicano sólo hasta el amparo, cuestión que retrasaba la solución final de los conflictos por causas del diseño institucional.

Otro de los temas importantes que contiene la reforma de 2008 es la constitucionalización de los mecanismos alternativos de solución de controversias como forma de solución de conflictos que se persiguen por la vía penal, principalmente para conductas que pudieran ubicarse dentro de los delitos patrimoniales. Los mecanismos alternativos permiten que el sistema de justicia tenga un escape temprano, previo a la formalización de los procesos jurisdiccionales. Con esto, las personas pueden acudir a las instituciones de justicia para resolver sus conflictos sin tener la necesidad de formalizar un proceso de litigio. Los mecanismos alternativos permiten también alterar la visión decimonónica de la justicia, a través de la cual los conflictos deberán resolverse mediante tribunales y leyes previamente establecidos, para permitir formas de composición más amigables y menos formales.

E1 29 de julio de 2010 se reformó el párrafo tercero del artículo 17 constitucional, para incluir las acciones colectivas como competencia de los jueces federales. La idea de la reforma es proveer un medio jurisdiccional para que se puedan proteger y restituir derechos de una colectividad mediante un solo proceso. Las acciones colectivas tradicionalmente se dirigen a ámbitos tales como la protección de los derechos del consumidor de bienes o servicios públicos o privados, servicios financieros y cuestiones medioambientales, pero no se limitan a ellos, sino que pueden extenderse a todo campo en donde pueda protegerse el interés de una colectividad. ${ }^{34}$ El centro de las acciones colectivas se encuentra en la capacidad de tutelar derechos que se ejercen de forma colectiva dentro de un solo proceso, lo que permite mayor claridad tanto en el ámbito material como subjetivo de la acción procesal.

La reforma más importante que ha tenido nuestra Constitución en sus más de cien años de vigencia sin duda es la del 10 de junio de 2011, conocida como la reforma en derechos humanos. Mediante ella no es exagerado señalar que se cambió la esencia del derecho constitucional mexicano. La Constitución reconoce a partir de entonces los derechos humanos de

\footnotetext{
34 Guidi, Antonio, Tutela de derechos difusos colectivos. Hacia un código modelo para Iberoamerica, México, Porrúa, 2004.
} 
fuente nacional e internacional, en lo que se llama el "parámetro de control de la regularidad constitucional", así como nuevos principios interpretativos tales como el principio pro persona y la interpretación conforme. Muy importante también, es que establece una obligación para todas las autoridades del país en la promoción, respeto protección y garantía de los derechos humanos.

Esta obligación en el ámbito jurisprudencial ha sido interpretada por la Suprema Corte como un deber ex officio de aplicar un control de constitucionalidad difuso para todos los juzgadores del país. ${ }^{35}$ Esto quiere decir que se cambia el paradigma anterior, mediante el cual el control de constitucionalidad correspondía en exclusiva a los tribunales federales del país, para obligar ahora a todos y cada uno de los órganos jurisdiccionales a ejercer un control de constitucionalidad ex officio en su labor.

Las implicaciones que tiene esta reforma para el control de la constitucionalidad en México son muy profundas, y vale la pena detenerse un momento en ellas. Tradicionalmente los jueces ordinarios estaban limitados en sus fuentes a la ley y la jurisprudencia, por lo que tenían que renunciar a realizar interpretación de la Constitución en su labor, dejando ésta a los tribunales federales. Esto provocaba que aun cuando el juez ordinario advirtiera la violación a un derecho o una evidente inconstitucionalidad, estaba impedido para decidir con base en ella, porque el ámbito de las fuentes jurídicas a su disposición no incluía la interpretación constitucional. La Constitución mandataba que únicamente correspondía a los tribunales federales dicha acción. De esta forma, un problema de naturaleza constitucional violatorio de derechos humanos podría posponerse para ser abordado por la vía del amparo judicial sólo después de la resolución que pusiera fin en definitiva al juicio.

Con la reforma constitucional al párrafo tercero, es fácil apreciar que el control de constitucionalidad difuso les da un espacio nuevo de acción para corregir problemas de violación de derechos humanos que puedan ad-

35 Expediente Varios 912/2010. Inicialmente el control de constitucionalidad difuso ex officio fue entendido por la Suprema Corte de Justicia de la Nación como la obligación de inaplicar una ley inconstitucional por todos los juzgadores del país. Posteriormente, el máximo tribunal ha trabajado sus criterios en casos posteriores, como la Contradicción de tesis 293/2011 y 22/2011, en donde ha continuado el análisis de las implicaciones de esta labor para llegar a la conclusión de que los juzgadores deben conocer el parámetro de regularidad constitucional en que se sitúa un caso. Véase Contradicción de tesis 293/2011 y Contradicción de tesis 20/2011. 
vertirse desde momentos muy tempranos del proceso, sin necesidad de la eventual intervención del amparo judicial. Al estar señalado el control de constitucionalidad difuso ex officio como una obligación, debemos considerar que es en este nivel en donde se encuentra la primera oportunidad de corregir violaciones a los derechos humanos, pero además, es ésta la más importante. Así, el amparo judicial deja de ser la única vía para el control de violaciones a los derechos humanos en el proceso judicial para ceder al juez ordinario la facultad de advertir violaciones por sí mismo.

El más reciente episodio del proceso de reforma judicial se produjo entre los años 2015 y 2017 mediante los denominados "Diálogos por la Justicia Cotidiana", una consulta y discusión pública a iniciativa tripartita del gobierno federal, el Centro de Investigación y Docencia Económicas y el Instituto de Investigaciones Jurídicas de la UNAM, mediante la cual se realizaron foros con la ciudadanía y mesas de análisis de los resultados con especialistas. ${ }^{36}$

Entre las nueve mesas temáticas que se formaron, la relativa a "Amparo y Resolución del Fondo del Conflicto" concluyó sus trabajos señalando que los problemas más relevantes del juicio de amparo en la actualidad pueden dividirse en cuatro categorías: "1) el uso excesivo del juicio de amparo para resolver temas que no son propiamente de justicia constitucional; 2) limites formales en el acceso al juicio de amparo; 3) deficiencias formales en el proceso, y 4) deficiencias en el uso e interpretación de los nuevos principios por parte del Poder Judicial". 37

Estas mesas de trabajo trajeron modificaciones importantes en dos rubros. El primero es en la reforma a la justicia laboral, para transitar de un paradigma procesal fundado en la conciliación y el arbitraje o la jurisdicción plena en la materia. Esta reforma es sumamente importante, debido a que los casos de amparo judicial en materia laboral inundan el trabajo de los tribunales colegiados de circuito año con año. El segundo, y que ha sido poco advertido por la academia en general, es el principio de resolu-

\footnotetext{
36 De acuerdo con los Diálogos por la Justicia Cotidiana, la justicia cotidiana "es la justicia mas cercana a las personas. La que vivimos día a día en nuestras interacciones ordinarias, la que facilita la convivencia armónica y la paz social. Es la que reclaman vecinos, trabajadores, padres de familia y la que se vive en las escuelas". Presentación del documento "Diálogos por la Justicia Cotidiana", consultado en www.gob.mx/justiciacotidiana.
}

37 Diálogos por la Justicia Cotidiana. Diagnósticos conjuntos y soluciones, Presidencia de la República-CIDE-UNAM, Instituto de Investigaciones Jurídicas, 2016. 
ción de fondo del conflicto que se incluyó en el artículo 17 de la Constitución, y que obliga a las autoridades a privilegiar la solución del conflicto sobre formalismos procedimentales. ${ }^{38}$

\section{LA REFORMA AL AMPARO Y SUS IMPLICACIONES PARA EL AMPARO JUDICIAL}

El seis de junio de 2011 se dio una reforma constitucional sustantiva en la materia, en donde además se ordenó la emisión de una nueva Ley de Amparo, publicada en 2013. ${ }^{39}$ La modificación en materia de amparo arribó tarde al proceso de reforma judicial en México, lo que ha contribuido a reducir las expectativas de su impacto dentro del sistema de justicia que se tuvieron desde finales del siglo XX. ${ }^{40}$

Los cambios principales que se introdujeron mediante la reforma constitucional refieren a la ampliación de su competencia para incluir los derechos humanos contenidos en tratados internacionales, incorporar el interés legítimo y la defensa de derechos colectivos, la posibilidad de emitir declaratoria general de inconstitucionalidad para que la Suprema Corte de Justicia de la Nación pueda expulsar normas del sistema jurídico, el amparo contra particulares que efectúen actos competencia de la autoridad, el amparo adhesivo y diversas reformas procesales orientadas a reducir la dilatación de los juicios ordinarios mediante el amparo para efectos, entre los más relevantes. ${ }^{41}$

Es importante señalar como primer tema, que no todos los contenidos de la reforma de amparo tocan al amparo judicial. Esto puede ser difícil

\footnotetext{
38 Siempre que no se afecte la igualdad entre las partes, el debido proceso u otros derechos en los juicios o procedimientos seguidos en forma de juicio, las autoridades deberán privilegiar la solución del conflicto sobre los formalismos procedimentales. Decreto publicado en el Diario Oficial de la Federación el 15 de septiembre de 2017.

39 Decreto por el que se reforman, adicionan y derogan diversas disposiciones de los artículos 94, 103, 104 y 107 de la Constitución Política de los Estados Unidos Mexicanos, Diario Oficial de la Federación, México, 6 de junio de 2011, primera sección, pp. 2-6.

40 En el texto Hacia una Nueva Ley de Amparo (Zaldívar, Arturo, México, Porrúa, 2010 , tercera edición) se narran las diez principales propuestas del proyecto de nueva ley de amparo resultado de los seminarios llevados a cabo en 2000 en el Instituto de Investigaciones Jurídicas de la UNAM.

41 Para consultar al detalle la reforma puede consultarse el siguiente vínculo http:// dof.gob.mx/nota_detalle.php? codigo $=5193266 \&$ fecha $=06 / 06 / 2011$.
} 
de comprender, ya que al amparo mexicano hay que entenderlo como una federación de medios de control que incluye diversos procesos con sus propias reglas, orientados a diversos ámbitos de protección regulados por la misma ley. ${ }^{42}$

Con la reforma de 2011, el amparo dejó de ser el gran olvidado del proceso de reforma al sistema de justicia en México. Los cambios que introduce podemos agruparlos en cinco grandes rubros: 1) la ampliación de la competencia material; 2) la ampliación de la puerta de entrada; 3) la ampliación de la resonancia jurisprudencial; 4) nuevos criterios de selectividad, y 5) control del amparo dilatorio o temerario. ${ }^{43}$

El primer tema es la ampliación de competencia material del juicio de amparo. Dicha ampliación se da mediante el cambio al artículo 103 constitucional, en donde se sustituyó el término "garantías individuales" por el de "derechos humanos", en consonancia con la nueva redacción del artículo primero constitucional. De esta forma, el ámbito de protección del amparo se extiende de un universo limitado respecto de las garantías individuales que otorgaba la Constitución, al ámbito mucho más vasto de los derechos humanos reconocidos por la Constitución, a lo que la doctrina llama el "bloque de constitucionalidad", y que hoy la Suprema Corte interpreta como el parámetro del control de la regularidad constitucional.

La ampliación de la puerta de entrada tiene que ver con la manera en que el Poder Judicial de la Federación admite a trámite los amparos. Históricamente, uno de los temas más controvertidos del juicio de amparo fue el limitado reconocimiento de legitimación a los individuos debido a la obligación de promoverse a través del interés jurídico, identificado como derecho subjetivo. ${ }^{44}$ A partir de la reforma constitucional, se introdujo la posibilidad de que el amparo se promueva no solamente por el titular de un derecho subjetivo, sino por quien sostenga tener “...un interés legítimo individual o colectivo, siempre que alegue que el acto reclamado viola los derechos reconocidos por esta Constitución y con ello se afecte su esfera

\footnotetext{
42 Fix-Zamudio, Héctor, Estudio de la defensa de la Constitución en el ordenamiento mexicano, México, Porrúa, 2011).

43 Existen otras clasificaciones importantes. Por ejemplo, Ferrer y Sánchez Gil mencionan a los siguientes como los cambios más relevantes que introduce la reforma: 1) tratados internacionales; 2) declaratoria general de inconstitucionalidad; 3) suspensión ponderativa; 4) amparo directo adhesivo, 5) plenos de circuito. Ferrer y Sánchez Gil, 2013.
}

\footnotetext{
44 Zaldívar, op. cit.
} 
jurídica, ya sea de manera directa o en virtud de su especial situación frente al orden jurídico".

La nueva redacción permite una puerta de entrada más amplia, dado que incluye no solamente la noción de un derecho subjetivo personal y directo, sino que se ensancha para hacerse colectivo, e incluye supuestos en donde el quejoso no sea necesariamente titular de un derecho subjetivo, sino de un derecho de naturaleza difusa. Pese a lo anterior, es importante observar que para el caso del amparo judicial dicha regla no tiene aplicación, y se mantiene el criterio procedimental del interés jurídico para los amparos contra sentencias que ponen fin a un juicio provenientes de tribunales judiciales, administrativos o de trabajo.

El caso de la ampliación de la resonancia jurisprudencial del trabajo del Poder Judicial de la Federación es otro punto destacado de la reforma. La fórmula Otero establece que los efectos de la sentencia en un juicio de amparo se limitan a las partes del litigio, es decir, que la inconstitucionalidad de un acto de autoridad o una norma decretada por un juez o tribunal federal solamente tiene efectos para quienes participen en el juicio. La reforma constitucional mantiene esta fórmula, pero ofrece una válvula de escape para que los efectos que producen las sentencias puedan tener mayor resonancia, a través de un novedoso proceso de creación de jurisprudencia de efectos erga omnes, denominado "declaratoria general de inconstitucionalidad" para el caso del amparo contra leyes.

En el caso del amparo judicial, no existe la posibilidad de acceder a la declaratoria general de inconstitucionalidad. La reforma, sin embargo, incluye la posibilidad de que las resoluciones tengan mayor resonancia y no se limiten a las partes del juicio mediante la figura del amparo adhesivo. Esta figura permite que otros sujetos con interés jurídico similar a los de las partes que intervinieron en el juicio puedan beneficiarse de una sentencia mediante la adhesión al amparo principal, siguiendo la misma suerte procesal que este.

La reforma de amparo de 2011 introdujo nuevos criterios de selectividad para la resolución de contradicción de tesis, al crear a los plenos de circuito como organismos intermedios entre los tribunales colegiados de circuito y la Suprema Corte de Justicia de la Nación. Conforme a la fracción XIII del artículo 107, cuando los tribunales colegiados de un mismo circuito sustenten tesis contradictorias, se puede promover denuncia de la contradicción ante el tribunal correspondiente. Si bien esta nueva facultad va más orientada a la organización de jurisprudencia formal dentro del Poder 
Judicial de la Federación, tiene un impacto positivo indirecto en el amparo judicial, debido a que los criterios formales que toman los tribunales colegiados de circuito en casos reiterados pueden someterse al control de un órgano intermedio antes de llegar a la Suprema Corte de Justicia, que son obligatorios para todos los juzgadores del país, y en muchos casos muy necesarios para orientar su labor.

Respecto a los límites al amparo dilatorio o temerario, la reforma busca desincentivar una práctica común entre los abogados de retrasar el fin de los juicios y la resolución definitiva de la sentencia mediante la interposición de amparos, muchas veces temerarios y sin fundamento, que provocan litigios interminables. Las medidas tomadas al respecto dentro del amparo judicial son varias, entre ellas limitar la suspensión en materias civil, mercantil y administrativa para obligar a garantizar por el quejoso los daños y perjuicios que pudiera ocasionar al tercero interesado o, en su caso, pedir al tercero interesado una contragarantía para asegurar la reposición de las cosas al estado que guardaban si se concediera el amparo.

En cuanto a violaciones acontecidas durante el procedimiento que se quieran hacer valer en amparo directo, se convierte en criterio de procedencia que las mismas hayan sido impugnadas durante la tramitación del juicio mediante el recurso o medio de defensa que señala la ley respectiva, con excepción de algunas personas vulnerables, como menores, incapaces $\mathrm{y}$ algunos otras.

Las reformas al juicio de amparo levantaron expectativas moderadas de una mejor justicia en México mediante una mayor protección de los derechos humanos de la población. Los cinco ejes principales en los que he explicado la reforma al juicio de amparo en relación con el amparo judicial contienen argumentos favorables para pronosticar un impacto positivo en el uso del amparo por las razones expuestas. Pese a lo anterior, también es importante tener presentes los límites que tiene la reforma, pues muestran que es aún incompleta, y que varios de los temas de mayor tensión permanecen presentes.

Algunos de los autores que han analizado el proceso de reforma muestran su descontento con partes del resultado. Pou Giménez señala que entre los problemas de la nueva Ley de Amparo están: que mantiene muchas limitaciones técnicas, como los plazos de interposición, la obligación de identificar a todas las autoridades potencialmente responsables, el incremento de las causales de improcedencia, la regulación de la suspensión 
(farragosa e incomprensible), la ausencia de medidas cautelares diferentes, los procesos tradicionales de ejecución y la ausencia de un lenguaje ciudadano. $^{45}$

Algunas demandas añejas quedaron fuera de la reforma de amparo, por lo cual no puede considerarse el proceso completo, y subsiste una conspicua agenda pendiente. Entre los temas más relevantes que no se modificaron destaca la permanencia de la concentración del juicio de amparo judicial como materia exclusiva de los tribunales federales, a pesar de que varias voces, entre ellas el Libro Blanco de la Reforma judicial, establecieron como una de sus prioridades la expansión de la competencia del amparo hacia los tribunales locales. Tampoco se dio una reforma procesal sustantiva que permitiera quitarle al amparo su carácter técnico especializado y hacerlo más accesible a la población. ${ }^{46}$

El poder reformador mexicano ha sido tímido en darle al amparo una identidad más ciudadana en la protección de sus derechos humanos, cuando en otros países de América Latina la reforma judicial ha traído, fortalecido o innovado medios accesibles de protección. Pero, por otro lado, le asigna una importancia alta, debido a que el juicio de amparo judicial continúa siendo la bisagra cardinal entre los sistemas jurisdiccionales federal y locales. Frecuentemente se encuentra en una tensión entre servir como un medio de protección de derechos humanos para el ciudadano y un medio especializado de control de la constitucionalidad de todo el sistema de justicia.

A pesar de los llamados a que el juicio de amparo se simplifique, hasta el momento el legislador no ha tomado esa dirección en el amparo en general, y tampoco en el amparo judicial. La ausencia de medios idóneos de los derechos que por mucho tiempo sufrió el sistema de justicia mexicano, junto con la tendencia a pensar al amparo como un remedio para todos los

45 Pou Giménez, Francisca, "El nuevo amparo mexicano y la protección de los derechos: ¿ni tan nuevo ni tan protector?”, Anuario de Derechos Humanos, núm. 10, Santiago, 2014, pp. 91-103.

46 Fabián Aguinaco sostiene que "independientemente de lo persuasiva que pudo ser la exposición de motivos relativo a que con la nueva Ley de Amparo se propone «eliminar tecnicismos y formalismos que dificultan el acceso al juicio de amparo», esta aseveración tiene poca fuerza". Aguinaco Bravo, Fabián María, "El juicio de amparo en el Centenario de la Constitución mexicana de 1917. Pasado presente y futuro", en Ferrer Mac-Gregor y Herrera García, Alfonso, El jucio de amparo en el centenario de la Constitución mexicana de 1917. Pasado, presente y futuro, t. I, México, UNAM, Instituto de Investigaciones Jurídicas, 2017. 
males, fortaleció la tesis de que el amparo debería tener un impacto directo en la justicia ordinaria, sobre todo por la gran desconfianza que existe respecto de la capacidad, probidad y eficiencia de las autoridades locales de seguridad y justicia. Para muchos, ha sido una decepción que el amparo no se modificara para ser más accesible a la población, mediante diversas estrategias; por ejemplo, ampliar la competencia a los tribunales locales. Por lo tanto, permanece como una tensión importante saber si el amparo mexicano finalmente consigue configurarse como el recurso judicial efectivo del cual hablan la Declaración Universal de los Derechos Humanos y la Convención Americana de los Derechos Humanos en sus artículos 8 y 25 para la protección de los derechos humanos.

Pese a lo anterior, no debe minimizarse su importancia como controlador del sistema de justicia. En el proceso de modificaciones constitucionales se ha buscado la concurrencia de nuevos procesos de atención de conflictos, tanto jurisdiccionales como no jurisdiccionales, para brindar una diversidad de posibilidades de solución de problemas, más sencillas, eficientes y baratas, en donde el amparo judicial no es ni la primera ni la más deseable forma de resolución. La inclusión de novedosos medios dentro del sistema de justicia ha provocado que incluso varios de los principios procesales con que se han construido estos medios choquen con la estructura de control de constitucionalidad concebida originalmente para el amparo judicial.

En el caso de la reforma procesal penal, existe uno de los ejemplos más claros de las incompatibilidades procesales entre las formas propias de contención de la constitucionalidad en el amparo, frente sus propias formas de contención de la constitucionalidad. ${ }^{47} \mathrm{El}$ caso se presenta más que evidente para el amparo judicial en la aparición del juez de control como un verdadero juez garante de la constitucionalidad del proceso penal. Aún así, la misma Constitución ha resuelto estos problemas señalando la obligación que tienen los quejosos de acudir primero a los medios de solución de esas violaciones que ofrecen los propios procedimientos ordinarios, en lugar de reservarlos al juez constitucional.

Los ejemplos de medios previstos para resolver las fallas en el sistema de justicia se multiplican en los procesos ordinarios. Una situación similar acontece con la reforma en materia de acciones colectivas, que si bien re-

\footnotetext{
47 Ferrer, Eduardo y Sanchez Gil, Rubén, El nuevo juicio de amparo y el proceso penal acusatorio, México, UNAM, Instituto de Investigaciones Jurídicas-IFP, 2013.
} 
sultan en un proceso resuelto también por los tribunales federales, brinda otras posibilidades de acceso a la justicia, sobre todo porque los conflictos no se enderezan en contra de autoridades, sino en contra de sujetos particulares sin necesidad de convertir el tema en una violación de derechos humanos.

El caso más relevante se encuentra con el control de convencionalidad difuso ex officio, el más reciente medio de control de constitucionalidad reconocido por el sistema mexicano, que permite que los temas de constitucionalidad se resuelvan desde un momento procesal más temprano, sin necesidad de establecerse como un procedimiento en sí mismo, sino como una herramienta interpretativa de los juzgadores para poder brindar justicia desde las instancias iniciales mediante la aplicación de un parámetro de regularidad constitucional a los casos concretos.

Del mismo modo, debemos tener en cuenta los mecanismos alternativos para la resolución de controversias, que privilegian formas de composición, como la negociación y la construcción de acuerdos por encima de la judicialización de los conflictos, en una concepción distinta de las formas tradicionales de obtener justicia. $\mathrm{O}$ de la obligación, para todas las autoridades, de aplicar el principio de la resolución del fondo del conflicto desde el primer momento de atención del conflicto.

Aunque entre una buena parte del sector de abogados existe la noción de que el juicio de amparo judicial es el único y verdadero medio de acceso a la justicia disponible para el ciudadano, el sofisticado sistema de justicia mexicano actual lo considera más bien el último recurso, posterior a todos los nuevos medios capaces de resolver violaciones al debido proceso y a otros derechos humanos en la resolución de conflictos.

En el diseño institucional actual, como no existía en el pasado, contamos con un complejo sistema de instituciones para resolver los conflictos cotidianos, por lo cual al amparo se le deben otorgan funciones específicas que lo regresen a una verdadera función de control del sistema de justicia y aleja la necesidad de convertirse en sustituto. Esto permite revalorar el nivel de excepcionalidad del amparo dentro de la justicia mexicana para fortalecer a los medios de nueva creación, que tienen la posibilidad de un impacto positivo desde etapas iniciales del conflicto, de forma más económica y eficiente y así reducir nuestra "amparo-dependencia". 


\section{CONCLUSIONES}

Es cierto que la reforma al juicio de amparo de 2011 llegó tarde, por lo menos diez años después de que sus bases estaban bien cimentadas en la discusión académica y comparada. Ese plazo fue suficiente para hacer que el amparo perdiera centralidad en el diseño institucional del sistema de justicia. Su reforma no tuvo la centralidad que se pensó tendría por mucho tiempo; por ejemplo, la reforma de derechos humanos tomada unos días después cimbró con mayor fuerza al sistema de justicia mexicano. La reforma más importante que sufrió el amparo aparece en los cambios que se realizaron al sistema de justicia mexicano, más que a la modificación de su diseño institucional. La forma adecuada de ver al amparo judicial hoy es como un procedimiento que tiene como función principal organizar la miscelánea de opciones procesales con las que se cuenta en el sistema de justicia desde su lugar especial como medio de control de constitucionalidad formal.

Entender esta función controladora es difícil cuando regionalmente en América Latina se puso énfasis desde hace tiempo en la necesidad de contar con un recurso judicial efectivo como garantía del derecho humano a la justicia. Varios países de la región construyeron un modelo de juicios de tutela y amparo más flexibles que sus figuras procesales previas, así como cortes y tribunales en donde este recurso se facilita en su atención, procedimiento y acceso. ${ }^{48}$

El hecho de que el juicio de amparo sea de jurisdicción concentrada en los tribunales federales es la causa más importante de la situación descrita en el párrafo anterior, y realmente se ve complicado que esto pueda alcanzarse sin eventualmente eliminar la doble jurisdicción del sistema federal mexicano. Con los límites técnicos y el alto nivel de especialización del amparo judicial difícilmente será ese recurso judicial efectivo que exige el derecho internacional.

A pesar de esta situación, el amparo judicial sí puede ayudar a controlar la constitucionalidad de los diversos medios propuestos para la resolución de los conflictos, proveyendo los criterios e interpretaciones orientadores de la función sustantiva de resolución de conflictos cotidianos, tanto por autoridades jurisdiccionales como no jurisdiccionales. Desde esa pers-

\footnotetext{
48 Uprimny, Rodrigo et al., ¿Justicia para todos? Sistema judicial, derechos sociales y democracia en Colombia, Bogotá, Norma, 2006. 
pectiva, el impacto positivo que pueda tener el juicio de amparo para la justicia ordinaria es que efectivamente ayude a controlar el funcionamiento de las demás instituciones del sistema judicial ideadas para la resolución de los conflictos cotidianos de una forma más sencilla y eficiente conforme a los estándares de protección constitucional de derechos humanos.

Además, en el nuevo diseño institucional de la justicia mexicana es necesario dejar de pensar al amparo judicial como sustituto de las instituciones de justicia en México, sino más bien como controlador e impulsor de la vigencia de las nuevas figuras que ha traído el largo proceso de reformas: desde los mecanismos alternativos no jurisdiccionales, hasta los nuevos procesos orales en materias civil, mercantil, penal o laboral. Serían muy malas noticias si ante la nueva miscelánea de opciones de solución de conflictos el amparo judicial necesitara asumir funciones sustitutivas, ante la deficiencia en el funcionamiento de los nuevos procedimientos. Es necesario que el Poder Judicial de la Federación deje crecer al sistema de justicia orientando su crecimiento, pero ya no sustituyéndolo.

Para ello, es necesario que se fortalezca el control difuso de la constitucionalidad que tienen las instituciones de justicia desde 2011, como la clave para construir un sistema de justicia que dependa en menor medida del amparo judicial. El control de constitucionalidad difuso ex officio lo que permite es que los jueces ordinarios aprendan a orientar su labor bajo criterios de constitucionalidad; si cuentan con la ayuda del Poder Judicial de la Federación para esto será muy útil.

Apelando al artículo de Héctor Fix-Fierro al inicio de texto, en donde visualizaba las distintas opciones que podía seguir el amparo judicial en México, es posible advertir que en gran medida se mantuvo el statu quo. De las demás opciones, la impresión que deja la reforma de amparo en el tema es que también de cierta manera hubo un límite a la procedencia del amparo judicial para alejarlo del uso temerario y acercarlo a un uso más institucional. Las opciones de grandes cambios institucionales, como la creación de supremas cortes a nivel local o de una reforma radical, quedaron desechadas. Lamentablemente, no se ha visto una mejora sustancial en la eficiencia y calidad de los tribunales ordinarios (esto, claro, a falta de datos empíricos expresos) ni una mejora sustancial en la formación y el ejercicio profesional de los abogados, que fueron los otros derroteros que el autor sugirió.

Para el futuro, hay opciones de que la reforma se profundice y que finalmente se pudiera tener un recurso judicial efectivo a nivel local parecido al 
amparo judicial. En las mesas de Diálogos de Justicia Cotidiana, el último gran debate nacional sobre el sistema de justicia, una de las conclusiones que quedó fue "fortalecer a los poderes judiciales de las entidades federativas y analizar la viabilidad de crear un recurso local similar al amparo casación que desincentive el abuso del amparo directo para la resolución de juicios de jurisdicción local". ${ }^{49}$

Así pues, no sobra recalcar que el contexto de la reforma en materia de amparo se acompaña de otras reformas (derechos humanos, mecanismos alternativos de solución de controversias, oralidad, tribunales laborales, etcétera) que se dirigen a fortalecer el sistema de justicia ordinaria, a través de los mecanismos institucionales expresamente diseñados para ello. Siendo así, la función actual del amparo judicial se reafirma como controladora del sistema de justicia, es decir, del amparo como forma de custodiar el buen desarrollo del sistema de justicia. En esta perspectiva, es importante observar sobre todo la relación que tiene el amparo con el control de los nuevos mecanismos con que cuenta el sistema de justicia, jurisdiccionales o no jurisdiccionales, traídos por la vasta y, hasta el momento, inconclusa agenda de reforma constitucional.

\section{BIBLIOGRAFÍA}

Aguinaco Bravo, Fabián María, "El juicio de amparo en el centenario de la Constitución mexicana de 1917. Pasado presente y futuro", en FERRER Mac-Gregor y HERRERA GARCÍA, Alfonso, El jucio de amparo en el centenario de la Constitución mexicana de 1917. Pasado, presente y futuro, t. I, México, UNAM, Instituto de Investigaciones Jurídicas, 2017. AnsolabeHERE, Karina, La política desde la justicia. Cortes supremas, gobierno y democracia en Argentina y México, México, Fontamara, 2007.

ANSOlABeHERE, Karina, Cambios en la política y cambios en la justicia: giro a la izquierda y Poder Judicial en América Latina, México, Fundación Friedrich Ebert en México, 2007.

BeER, Caroline C., "Judicial Performance and the Rule of Law in the Mexican States", Latin American Politics and Society, vol. 48, Issue 3 September, 2006.

49 Diálogos por la Justicia Cotidiana. Diagnósticos conjuntos y soluciones, Presidencia de la República-CIDE-UNAM, Instituto de Investigaciones Jurídicas, 2016, p. 234) 
Brage CAMAZANO, Joaquín, La acción abstracta de inconstitucionalidad, México, UNAM, Instituto de Investigaciones Jurídicas, 2005.

Bustillos, Julio, Federalismo judicial a través del amparo, México, UNAM, Instituto de Investigaciones Jurídicas, 2010.

CABAllero JuÁREZ, José Antonio et al., Libro Blanco de la reforma judicial en México, México, SCJN, 2006.

Cossío DíAz, José Ramón, Cambio social y cambio jurídico, México, Miguel Ángel Porrúa-ITAM, 2001.

Domingo, Pilar "Rule of Law, Citizenship and Access to Justice in Mexico", Mexican Studies/Estudios mexicanos, vol. 15, núm. 1, Winter 1999.

FERRER MAC-GREGOR, Eduardo, "Hacia un derecho procesal constitucional local en México", Anuario de Derecho Constitucional Latinoamericano, 2003, Montevideo, Konrad Adenauer Stiftung, 2003.

FERrer MAC-Gregor, Eduardo y SÁNCHEZ GIL, Rubén, El nuevo juicio de amparo y el proceso penal acusatorio, México, UNAM, Instituto de Investigaciones Jurídicas-IFP, 2013.

FERrer MAC-Gregor, Eduardo y VEGA HERNÁNDEZ, Rodolfo (coords.) Justicia constitucional local, Querétaro, FUNDAP-Gobierno del Estado de Coahuila, 2002.

FERRER, Eduardo y ZALDIVAR, Arturo (coords), La ciencia del derecho procesal constitucional: Estudios en homenaje a Héctor Fix-Zamudio en sus cincuenta años como investigador del derecho, t. II, México, UNAM, Instituto de Investigaciones Jurídicas, 2009.

FIX-FIERRO, Héctor, "Entre la eficacia autoritaria y la incertidumbre democrática”, en PÁSARA, Luis (comp.), En busca de una justicia distinta. Experiencias de reforma en América Latina, México, UNAM, 2009.

FIX-FIERRO, Héctor, Tribunales, justicia y eficiencia. Estudio sociojuridico sobre la racionalidad económica en la función judicial, Mexico, UNAM, Instituto de Investigaciones Jurídicas, 2006.

FIX-FIERRO, Héctor, La reforma judicial mexicana, ¿de dónde viene? ¿hacia dónde va?, México, UNAM, Instituto de Investigaciones Jurídicas, 2002.

FIX-FIERRO, Héctor et al., Entre un buen arreglo y un mal pleito, Encuesta Nacional de Justicia, México, UNAM, 2015. 
FiX-ZAmudio, Héctor, Estudio de la defensa de la Constitución en el ordenamiento mexicano, México, Porrúa, 2011.

LARA, Roberto et al., “¿De verdad deseamos una Corte redentora?”, $\mathrm{Ne}$ xos, núm. 372, diciembre de 2008.

LOPEZ AYLLÓN, Sergio y FIX-FierRo, Héctor, “¡Tan cerca, tan lejos! Estado de Derecho y cambio jurídico en México (1970-2000)", Boletín Mexicano de Derecho Comparado, México, año XXXIII, núm. 97, enero-abril de 2000.

MAGALONI, Ana Laura, “¿Por qué la Suprema Corte no ha sido instrumento para los derechos fundamentales?", en FERRER, Eduardo y ZALDIVAR, Arturo, La ciencia del derecho procesal constitucional. Estudios en homenaje a Héctor Fix-Zamudio en sus cincuenta años como investigador del derecho, t. II, México, UNAM, Instituto de Investigaciones Jurídicas, 2009.

MAGALONI, Ana Laura y ZALDÍvAR, Arturo, "El ciudadano olvidado", $\mathrm{Ne}$ xos, México, año 28, vol. XXVIII, núm. 342, junio de 2006.

PÁSARA, Luis (comp.), En busca de una justicia distinta. Experiencias de reforma en América Latina, México, UNAM, 2009.

PÉrez Perdomo, Rogelio, Gente del derecho y cultura jurídica en América Latina, México, UNAM, Instituto de Investigaciones Jurídicas, 2013.

Pou GiMÉnEZ, Francisca, "El nuevo amparo mexicano y la protección de los derechos: ¿ni tan nuevo ni tan protector?”, Anuario de Derechos Humanos, núm. 10, Santiago, 2014.

Rios FigueroA, Julio, "Justicia constitucional y derechos humanos en América Latina” Revista Latinoamericana de Política Comparada, vol. 3, enero de 2010.

Rios FigueroA, Julio, "Fragmentation of Power and the Emergence of an Effective Judiciary in Mexico", Latin American Politics and Society, Spring, vol. 49, num. 1, 2007.

SuAREZ ÁvilA, Alberto Abad, La protección de los derechos fundamentales en la Novena Época de la Suprema Corte, México, Porrúa, 2015.

TAFOYA HERnÁNDEZ, J. Guadalupe (coord.), Elementos para el estudio del juicio de amparo, México, SCJN, 2017.

TAMAYO SALMORÁN, Rolando, "El poder y la judicatura (breve comentario sobre la jurisdicción de amparo y la función judicial”, Boletín Mexi- 
cano de Derecho Comparado, núm. 63, México, UNAM, Instituto de Investigaciones Jurídicas, 1998.

TAte, C. Neal y VAlLinder, Torbjörn (eds.), The Global Expansion of Judicial Power, New York, New York University Press, 1995.

Tortolero Cervantes, Francisco y Pérez VÁzQuez, Carlos (coords.), El juicio de amparo en la Declaración Universal de los Derechos Humanos, México, SCJN, 2014.

UPRIMNY, Rodrigo et al., ¿Justicia para todos? Sistema judicial, derechos sociales y democracia en Colombia, Norma, 2006.

ZALDÍVAR, Arturo, Hacia una nueva Ley de Amparo, México, UNAM, Instituto de Investigaciones Jurídicas, 2002.

Zaldívar, Arturo, Diálogos por la Justicia Cotidiana. Diagnósticos conjuntos y soluciones, Presidencia de la República-CIDE-UNAM, Instituto de Investigaciones Jurídicas, 2016.

Fecha de recepción: 20 de noviembre de 2018.

Fecha de aceptación: 14 de diciembre de 2019. 